\title{
Methodologie zur Optimierung von Geschäftsprozessen
}

\author{
Klaus D. Bösing
}

\section{Einleitung}

Wie aus Fachpublikationen und Gesprächen mit der Industrie bekannt, stehen zurzeit die Geschäftsprozessmodellierung und Geschäftsprozessoptimierung im Mittelpunkt von Forschungs- und Entwicklungsaktivitäten. Derzeit sind viele Unternehmen damit beschäftigt, ihre Geschäftsprozesse zu überdenken und neu zu strukturieren, um am Markt erfolgreich zu sein.

Im Folgenden soll eine Methodologie entwickelt werden, mit der sich aufgrund von Leistungsindikatoren Geschäftsprozesse analysieren und bewerten lassen. Dazu ist eine Abgrenzung zwischen Leistungsindikatoren zur Bewertung von Geschäftstätigkeiten (Key Performance Indicator) und den der Geschäftstätigkeiten zugrunde liegenden Geschäftsprozesse (Process Performance Indicator) vorzunehmen. Mithilfe einer Methodik zur Generierung dieser geschäftsprozessbezogenen Leistungsindikatoren sollen Möglichkeiten geschaffen werden, aufgrund von quantitativen und qualitativen Aussagen eines Geschäftsprozesses Rückschlüsse auf die Qualität dieses Geschäftsprozesses schließen zu können. Die Optimierung der Geschäftsprozesse im Sinne eines kontinuierlichen Verbesserungsprozesses hat einen direkten Einfluss auf den Erfolg eines Unternehmens.

\subsection{Einführung in die Thematik}

Nur wer sich dem schnellen Wandel in globalisierten Märkten ständig anpasst, ist auch erfolgreich. Konsequente Kostenoptimierung und maximale Kundenorientierung sind dafür wichtige Voraussetzungen. In diesem Zusammenhang gewinnen Geschäftsprozesse immer mehr an Bedeutung. Dabei geht es nicht nur um die Automatisierung vorhandener Prozesse, sondern auch darum, Veränderungen frühzeitig zu erkennen und schnell zu reagieren. „Prozessdenken ist für IT-Manager künftig überlebenswichtig“, so der Analyst der Gartner Group, Inc. „Eine ihrer Hauptaufgaben wird darin bestehen, operative Geschäftsabläufe von Anfang bis Ende zu unterstützen und gleichzeitig das Management zeitnah über Veränderungen von Schlüsselindikatoren zu informieren" [4].

Wie beim Konzept der Business Prozess Fusion, dem Dokumentenmanagement, dem Systemmanagement, dem Applikationsmanagement, den Archivsystemen, dem Projektmanagement, um nur einige Beispiele zu nennen, haben Geschäftsprozesse bzw. Geschäftsprozessoptimierungen oberste Priorität.

Ein Geschäftsprozess ist eine Reihe von aufeinander folgenden Tätigkeiten oder Aktivitäten, um eine Dienstleistung oder ein Produkt zu planen, zu produzieren und zu vertreiben. Geschäftsprozesse lassen sich in drei Hauptkategorien untergliedern:

\section{- Führungsprozesse}

- Kernprozesse

- Unterstützungsprozesse

Welche Prozesse wie durchzuführen sind, damit sie effektiv und effizient ablaufen, damit das Ergebnis mit möglichst wenig Ressourcen und geringen Kosten erreicht wird, lässt sich mithilfe von Geschäftsprozessmanagement realisieren. „Unter Geschäftsprozessmanagement wird ein integriertes Konzept von Führung, Organisation und Controlling verstanden, das eine zielgerichtete Steuerung der Geschäftsprozesse ermöglicht und das gesamte Unternehmen auf die Erfüllung der Bedürfnisse der Kunden und anderer Interessengruppen (Mitarbeiter, Kapitalgeber, Eigentümer, Lieferanten, Partner, Gesellschaft) ausrichtet“ [18].

Bei der Einführung des Geschäftsprozessmanagements sind insbesondere Geschäftsprozessmodelle von elementarer Bedeutung, um Geschäftsprozesse angemessen zu beschreiben, damit sie analysiert, optimiert und automatisiert werden können. Damit ist die Geschäftsprozessmodellierung ein wesentlicher Bestandteil des Geschäftsprozessmanagements.

Ein weiterer essenzieller Bestandteil des Geschäftsprozessmanagements ist die Optimierung von Geschäftsprozessen. Bei der Geschäftsprozessoptimierung werden zwei grundsätzliche Ansätze verfolgt:

- der behutsame Ansatz, auch als empirische Vorgehensweise bezeichnet, mit der über kontinuierliche Verbesserungen die Leistungsfähigkeit der Geschäftsprozesse erhöht wird,

- der konzeptionelle Ansatz, der radikale Weg des Business Process Reengineering nach M. Hammer und J. Champy [11]. Dieser Ansatz besteht aus einer umfassenden Neugestaltung ausgewählter Geschäftsprozesse.

Die elementare Frage, die sich mit der Optimierung von Geschäftsprozessen stellt, ist, wie man die Qualität von Geschäftsprozessen misst. Die entscheidenden Faktoren sind hier die Bestimmung, Messung und Bewertung von Leistungsgrößen, die zum Erreichen der Ziele eines Unternehmens führen sollen. Diese Leistungsmessung wird unter dem Begriff Performance Management zusammengefasst.

Das wohl bekannteste Konzept zur Messung der Leistungen ist der so genannte Balanced Scorecard-Ansatz von Kaplan und Norton [15], der auch überwiegend als Basis für zahlreiche Software-Adaptionen des Performance Management dient. Ziel der Balanced Scorecard ist es, mit ausgewählten Kenngrößen das Unternehmen strategiekonform zu steuern. Grundlage der Balanced Scorecard sind Messkriterien, d. h. Messgrößen einzelner Zielkategorien der

- Finanzperspektive

- Kundenperspektive

- interne Prozessperspektive

- Lern- und Entwicklungsperspektive (vgl. u. a. [14]) 
Hinsichtlich der Darstellung von Zielen lassen sich diese weiter verfeinern, wie beispielsweise in so genannte Prozesskennzahlen oder Leistungsindikatoren (Key Performance Indicator, Process Performance Indicator).

Um die Qualität von Geschäftsprozessen messen zu können, sind also entsprechende Leistungsindikatoren zu definieren. Hierzu ist es notwendig, die Qualität von Geschäftsprozessen systematisch $\mathrm{zu}$ erfassen und $\mathrm{zu}$ bewerten. Dabei stellt sich zunächst die Frage, was gemessen werden soll. Normalerweise ist es nicht möglich, die Qualität eines Geschäftsprozesses mit einer einzigen Kenngröße zu ermitteln. Um Aussagen über die Qualität von Geschäftsprozessen machen zu können, sind in der Regel viele verschiedene Kriterien und Messgrößen heranzuziehen und deren Ausprägungen zu messen. Die gemessenen Leistungsindikatoren lassen sich dann im Kontext der Geschäftsprozesse bewerten und interpretieren. Dies kann wiederum die Ausgangsbasis bilden, um Verbesserungsmaßnahmen einzuleiten.

\subsection{Konkretes Vorgehen}

Bei der Optimierung von Geschäftsprozessen stellt sich zunächst die Frage, wie man die Qualität von Geschäftsprozessen misst. Im Vorfeld ist daher zu klären,

- für welche Geschäftsprozesse Leistungsindikatoren entwickelt werden sollen,

- wie die zu messenden Geschäftsprozesse strukturiert sind,

- was in den Geschäftsprozessen abläuft,

- wer sich an den Geschäftsprozessen beteiligt und

- wie sich die Geschäftsprozessumgebung gestaltet.

Um diese Fragen zu beantworten, sind zunächst Geschäftsprozessmodelle für ausgewählte Geschäftsprozesse zu erstellen. Für die Modellierung von Geschäftsprozessen wird in der Praxis eine Vielzahl von Ansätzen vorgeschlagen. Für die praktische Umsetzung wird der Ansatz der ereignisgesteuerten Prozessketten angestrebt, weil dieser den aktivitätsorientierten Entwurf unterstützt und die logischen Abhängigkeiten zwischen den Aktivitäten veranschaulicht. Außerdem können den Aktivitäten die für die Ausführung zuständige organisatorische Einheit (Abteilung, Stelle etc.) zugewiesen werden.

Nach der empirischen Vorgehensweise müssen zunächst die ausgewählten Geschäftsprozesse hinsichtlich ihrer Ziele und Erwartungen formuliert werden, um die Qualität einzelner Geschäftsprozesse ermitteln zu können. Hierzu sind zunächst die Geschäftsprozesse und deren organisatorischen und technischen Rahmenbedingungen systematisch zu analysieren.

Bei der Zielsetzung liegt der Schwerpunkt auf der internen Prozessperspektive, eine der Sichtweisen der Balanced Scorecard. Mithilfe der Balanced Scorecard soll erreicht werden, die definierten Ziele der Geschäftsprozesse in einem ständig ablaufenden Prozess zu evaluieren.

Als nächstes stellt sich die Frage, welche Leistungsindikatoren eines Geschäftsprozesses relevant sind, um die definierten Ziele zu erreichen. Im Folgenden sollen hier die Leistungsindikatoren der Geschäftstätigkeiten zugrunde liegenden Geschäftsprozesse (Process Performance Indicators) betrachtet werden. Dabei wird ein Ansatz entwickelt, Leistungsindikatoren auf der Grund- lage von Erfolgsfaktoren abzuleiten. Erfolgsfaktoren sind Größen, die den Unternehmenserfolg entscheidend und nachhaltig beeinflussen. Bezogen auf die Ebene der internen Prozessperspektive liefern sie Auskunft über die Performance von Geschäftsprozessen. Hierzu müssen zunächst die Geschäftsprozess-Erfolgsfaktoren ermittelt werden, sodass die Geschäftsprozesse hinsichtlich der erfolgsrelevanten Faktoren gemessen werden können. Auf dieser Grundlage lassen sich dann Leistungsindikatoren für die entsprechenden Geschäftsprozesse definieren. Mithilfe dieser Leistungsfaktoren und des Ziels des Geschäftsprozesses lässt sich somit ein Soll-/Ist-Vergleich durchführen.

Um die Entscheidungen von Verbesserungsmaßnahmen nur auf wenige Einflussgrößen zu reduzieren, sollen die Leistungsindikatoren der Geschäftsprozesse der unterschiedlichen Dekompositionsebenen auf kausale Zusammenhänge hin analysiert werden, insbesondere, welche Ursache-Wirkungs-Zusammenhänge zwischen den Zielen und Erfolgsfaktoren der Geschäftsprozesse in unterschiedlichen Dekompositionsebenen bestehen, um geschäftsprozessbezogen Leistungsindikatoren zusammenfassen zu können.

\section{Definition von Leistungsindikatoren}

Bei der Optimierung von Geschäftsprozessen stellt sich die grundlegende Frage, wie man die Qualität von Geschäftsprozessen misst. Die entscheidenden Faktoren sind die Bestimmung, Messung und Bewertung von Leistungsgrößen, die zum Erreichen der Ziele eines Unternehmens führen sollen.

Eines der bekanntesten Konzepte zur Messung der Leistungen von Geschäftsprozessen ist die Balanced Scorecard von R. S. Kaplan und D. P. Norton (vgl. [15]). Ziel dieser Balanced Scorecard ist es, mithilfe von entsprechenden Leistungsindikatoren ein Unternehmen strategiekonform zu steuern.

Welche Leistungsindikatoren eines Geschäftsprozesses sind nun relevant, um die definierten Ziele zu erreichen?

Im Folgenden wird auf der Grundlage der Balanced Scorecard ein Ansatz entwickelt, um Leistungsindikatoren zur Bewertung von Geschäftstätigkeiten in Abgrenzung zu den der Geschäftstätigkeiten zugrunde liegenden Geschäftsprozesse auf der Basis von Erfolgsfaktoren abzuleiten. Erfolgsfaktoren sind Größen, die den Unternehmenserfolg entscheidend und nachhaltig beeinflussen.

\subsection{Balanced Scorecard}

Das Konzept der Balanced Scorecard beruht auf der Grundidee, die strategischen Ziele eines Unternehmens, hier am Beispiel der gedas AG [10], hinsichtlich der operativen Geschäfte durch die vier unterschiedlichen Perspektiven

- die Finanzperspektive,

- die Kundenperspektive,

- die Prozessperspektive und

- die Potenzialperspektive miteinander zu verbinden.

Nach R. S. Kaplan und D. P. Norton soll mithilfe dieser vier Perspektiven eine Balance zwischen lang- und kurzfristi- 
gen Zielen, monetären und nicht monetären Indikatoren, Früh- und Spätindikatoren sowie harten objektiven und weichen subjektiven Indikatoren abgeleitet werden. In der Finanzperspektive werden die Ziele eines Unternehmens beschrieben, die Auskunft geben über die finanzwirtschaftlichen Ziele wie Kapitalrendite, Umsatzwachstum und Kostensituation. Bei der Kundenperspektive steht der Kunde und seine Interessen im Mittelpunkt. Hier geht es in erster Linie um die Steigerung der Kundenzufriedenheit und wie diese umgesetzt werden kann. Die Prozessperspektive gibt Auskunft über die Leistung einzelner interner Geschäftsprozesse. Sie gibt an, welche Geschäftsprozesse welche Leistung erbringen müssen, damit die Finanz- und Kundenziele erreicht werden können. In der Potenzialperspektive werden beispielsweise Aussagen bezüglich der Entwicklung der Mitarbeiterpotenziale, der Infrastruktur und der Potenziale der Informationssysteme getroffen.

Ein wesentlicher Bestandteil der Balanced Scorecard ist, dass neben den Wirkungen (Finanz-Aspekt) auch die Ursachen (Kunden-Aspekt, Prozess-Aspekt, Potenzial-Aspekt) berücksichtigt werden. In diesem Zusammenhang spricht man auch von einer Ursache-Wirkungs-Kette (Abb. 1). Die einzelnen Perspektiven lassen sich als eine Rangfolge betrachten: Die Ziele der unteren Ebene (Potenzial- und Prozessperspektive) gelten als so genannte Leistungstreiber. Denn der Erfolg dieser Ebenen ist Bedingung dafür, um die Ziele der oberen Ebene (Finanzperspektive, Kundenperspektive) zu erreichen.

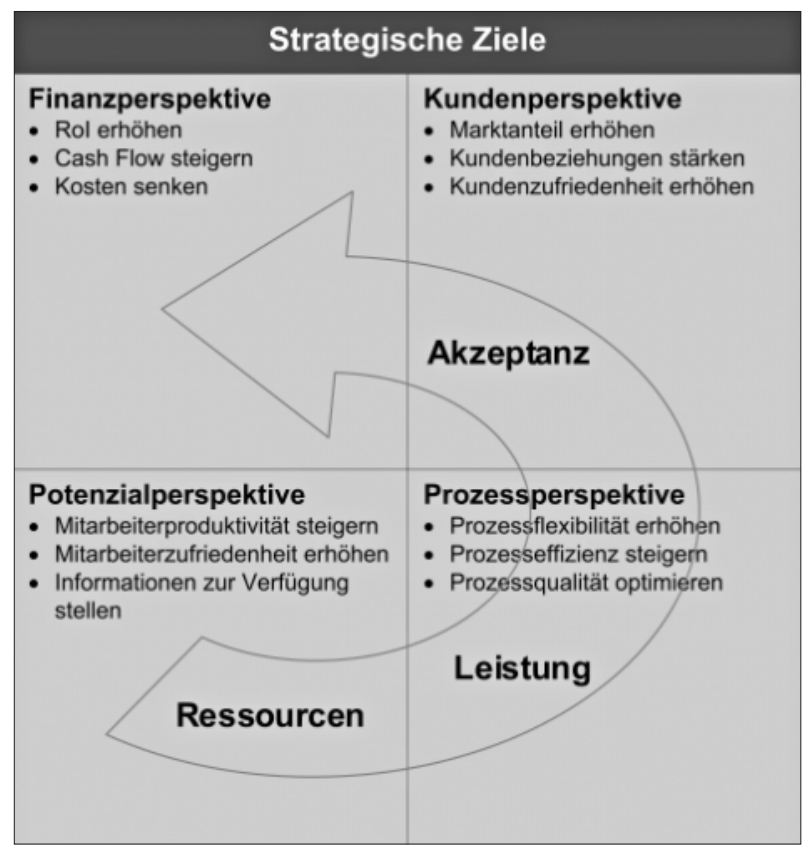

Abb. 1: Strategische Ziele und Perspektiven der Balanced Scorecard (vgl. [20])

Um die Ziele der einzelnen Perspektiven bewerten zu können, um letztendlich die Effektivität und Effizienz eines Unternehmens zu steigern, müssen entsprechende Messgrößen, so genannte Leistungsindikatoren, eingeführt werden. Denn nur so lassen sich Aussagen darüber treffen, ob die Ziele erreicht werden. Hierbei gilt es, sich auf wenige Indikatoren zu konzentrieren, die für die strategischen Ziele und somit für den Erfolg eines Unternehmens relevant sind.
Leider gibt es in einschlägigen Publikationen keine Hinweise bezüglich der Abgrenzung von Leistungsindikatoren, so dass zunächst eine Grundlage geschaffen werden muss, um die Indikatoren des Prozessmodells zu analysieren.

Wie lassen sich nun diese unterschiedlichen Indikatoren den zugrunde liegenden Zielkategorien und den Geschäftsprozessen zuordnen? Dazu soll im Folgenden eine Definition der entsprechenden Leistungsgrößen vorgenommen werden.

\subsection{Definition des Key Performance Indicators}

Der Key Performance Indicator (KPI) ist jener Indikator, der für das Ergebnis eines Geschäftsprozesses relevant ist. Dieser lässt sich aus den strategischen Zielen der Finanzperspektive, Kundenperspektive und Potenzialperspektive der Balanced Scorecard ableiten.

Beispielsweise lassen sich folgende KPIs aus den Zielen der entsprechenden Perspektiven ableiten:

- RoI

- Betriebsergebnis

- Cash Flow

- Kundenzufriedenheit

- Kundentreue

- Kundenbeziehungen

- Mitarbeiterproduktivität

- Mitarbeitertreue

- Mitarbeiterzufriedenheit Finanzperspektive

Da diese Indikatoren aus Publikationen und der Praxis hinreichend bekannt sind, wird an dieser Stelle auf weitere Erläuterungen verzichtet.

\subsection{Definition des Process Performance Indicators}

Der Process Performance Indicator (PPI) ist jener Indikator, der für die Güte eines Geschäftsprozesses relevant ist. Dieser lässt sich aus den strategischen Zielen der Prozessperspektive der Balanced Scorecard ableiten.

Die wichtigsten PPIs der Prozessperspektive sind hier die

- Prozesszeit,

- Prozesskosten,

- Prozessqualität und

- Termintreue (interne)

Die Prozesszeit eines Geschäftsprozesses setzt sich aus der Summe der Prozesszeiten aller Teilprozesse, der Durchlaufzeit zusammen (Abb. 2):

$P Z=P Z_{T P_{1}}+P Z_{T P_{2}}+\ldots+P Z_{T P_{n-1}}+P Z_{T P_{n}}$

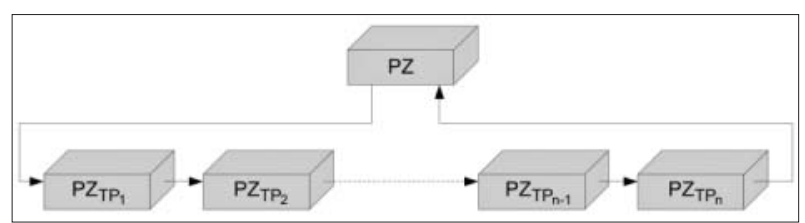

Abb. 2: Prozesszeit ohne parallele Prozesse

Finden logische Verknüpfungen in Form von parallelen Aktivitäten statt, so ist an dieser Stelle für die Prozesszeit die Zykluszeit zu berücksichtigen (Abb. 3, vgl. u. a. [18]):

$P Z=P Z_{T P_{1}}+P Z_{T P_{2}}+\ldots+P Z_{T P_{n-1}}+P Z_{T P_{n}}+\sum$ aller paralleler Prozesse 


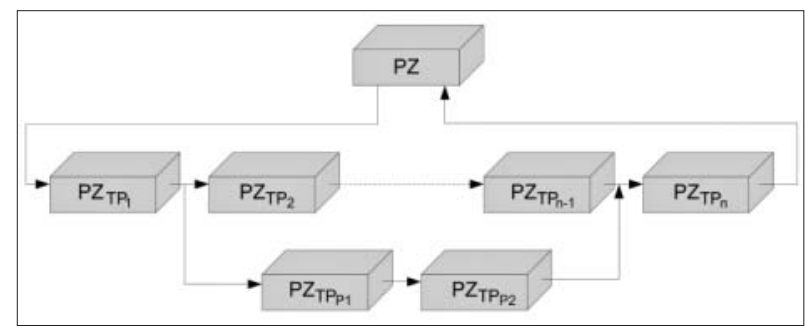

Abb. 3: Prozesszeit mit parallelen Prozessen

Beispielsweise ist für die Erstellung von Angeboten im Vertriebsprozess, für das Bearbeiten von Aufträgen im Auftragsabwicklungsprozess oder für die Beseitigung von Fehlern im Supportprozess die Durchlaufzeit der geeignete Indikator. Mithilfe der Zykluszeit lässt sich die Effizienz eines Geschäftsprozesses messen, da hier die Dauer der Ressourcenbindung zusätzlich zu der Bearbeitung eines Objekts Einfluss nimmt. Ferner lassen sich Prozesszeiten in statische und dynamische Prozesszeiten untergliedern, auf die an dieser Stelle nicht näher eingegangen werden soll.

Die Prozesskosten zeigen auf, welche Ressourcen ein Geschäftsprozess verbraucht und was die Erzeugung von Prozessleistungen kostet, wie beispielsweise die Erstellung eines Angebotes. Mit Prozesskosten werden Leistungen monetär bewertet und stellen somit eine Verbindung zwischen Prozessleistung, Ressourcenverbrauch und wirtschaftlichem Ergebnis her.

Die Prozesskosten setzen sich wie die Prozesszeit ebenfalls aus der Summe der Prozesskosten aller Teilprozesse zusammen (Abb. 4):

$P K=P K_{T P_{1}}+P K_{T P_{2}}+\ldots+P K_{T P_{n-1}}+P K_{T P_{n}}$

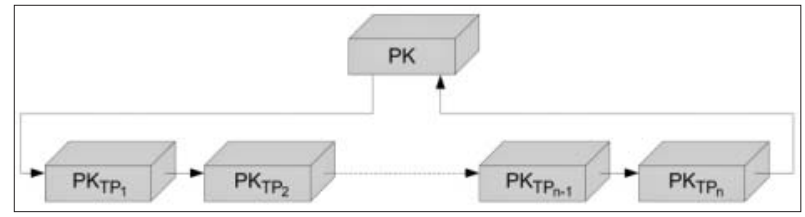

Abb. 4: Prozesskosten ohne parallele Prozesse

Handelt sich um parallele Aktivitäten, so sind hier für die Berechnung der Prozesskosten ebenfalls die parallelen Prozesse zu berücksichtigen.

Die Prozessqualität gibt Auskunft über die interne Zufriedenheit mit dem Prozessverlauf und den erzielten Ergebnissen eines Prozesses. Eine hohe Prozessqualität lässt auf eine hohe Prozesseffizienz und eine hohe Produktqualität schließen. Messgrößen sind hier First Pass Yield, die Fehlerrate und Fehlleistungskosten. Da die Aussagekraft der Fehlleistungskosten häufig an der ungenauen und unvollständigen Kostenerfassung und Kostenzurechnung leidet, wird diese an dieser Stelle nicht näher beschrieben (vgl. [18]).

Unter First Pass Yield (FPY) wird der Anteil der Prozessergebnisse verstanden, die bereits nach dem ersten Prozessdurchlauf korrekt sind und keine Nacharbeit erfordern (vgl. u. a. [18]). Auf der Grundlage des FPY wird die Prozessqualität wie folgt ermittelt:

$P Q=\frac{\sum \text { abgeschlossene Ergebnisse }\left(t_{0}-t_{-1}\right) \text { ohne Nachbearbeitung }}{\sum \text { aller abgeschlossenen Ergebnisse }\left(t_{0}-t_{-1}\right)} \times 100$

wobei $\left(t_{0}-t_{-1}\right)$ die Messperiode bedeutet.
PQ hat den Wert 0, wenn das Prozessergebnis nach dem ersten Prozessdurchlauf fehlerfrei ist, ansonsten den Wert 1 (Abb. 5).

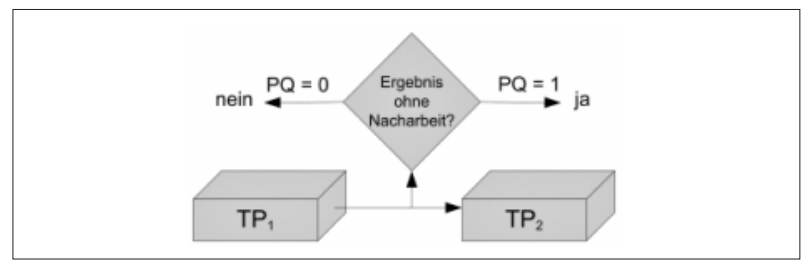

Abb. 5: Prozessergebnis nach dem ersten Durchlauf

Beispiele für fehlerhafte Prozessdurchläufe sind unvollständige oder fehlerhafte Angebote und Vertragskorrekturen.

Die Prozessqualität wird durch die Multiplikation der Prozessqualität der Teilprozesse ermittelt (Abb. 6):

$P Q=P Q_{T P_{1}} \times P Q_{T P_{2}} \times \ldots \times P Q_{T P_{n-1}} \times P Q_{T P_{n}}$

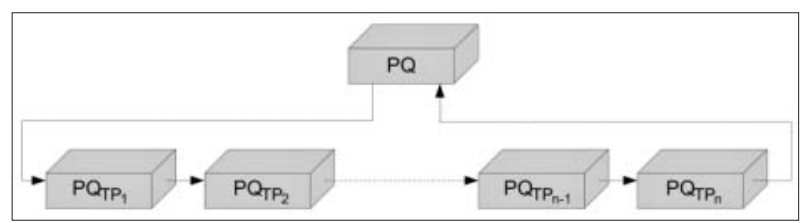

Abb. 6: Prozessqualität

Als Zielwert für die Prozessqualität sollten $100 \%$ erreicht werden.

Unter der Fehlerrate wird der Anteil der fehlerhaften Prozessergebnisse an der Gesamtheit der Ergebnisse verstanden, wobei Fehler als Abweichung vom Soll gesehen werden (vgl. u. a. [5]):

Fehlerrate $=\frac{\sum \text { über alle fehlerhaften Ergebnisse in }\left(t_{0}-t_{-1}\right)}{\text { Anzahl der Ergebnissein }\left(t_{0}-t_{-1}\right)}$

Während beim FPY die Trefferquote der Prozessergebnisse ermittelt wird, gehen bei der Fehlerrate die Zahl der Fehler direkt ein.

Die interne Termintreue gibt Auskunft darüber, wie hoch der Anteil der Ergebnisse ist, die der Prozess zum vorgesehen Termin ohne Terminverzögerung liefert (vgl. u. a. [18]):

$T T=\frac{\sum \text { fertiggestellen Ergebnisse }\left(t_{0}-t_{-1}\right) \text { ohneTerminverzögerung }}{\sum \text { aller fertiggestellten Ergebnissein }\left(t_{0}-t_{-1}\right)} \times 100(7)$

Die interne Termintreue hat den Wert 0, wenn das Prozessergebnis nach dem ersten Prozessdurchlauf ohne Terminverzögerung ist, ansonsten den Wert 1 (Abb. 7).

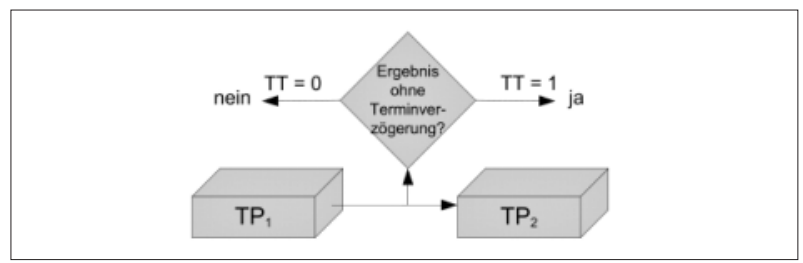

Abb. 7: Prozessergebnis nach dem ersten Durchlauf

Die Termintreue wird durch die Multiplikation der einzelnen Werte der Teilprozesse ermittelt (Abb. 8):

$T T=T T_{T P_{1}} \times T T_{T P_{2}} \times \ldots \times T T_{T P_{n-1}} \times T T_{T P_{n}}$ 


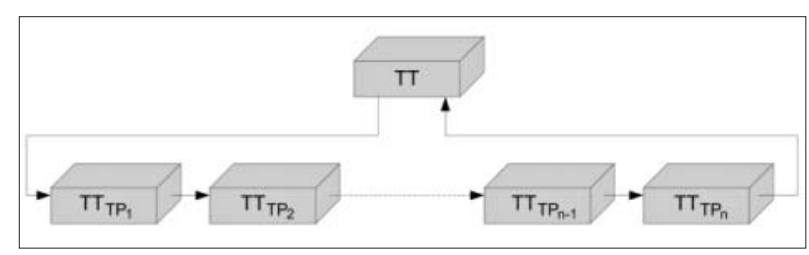

Abb. 8: Interne Termintreue

Als Zielwert für die interne Termintreue sollten auch $100 \%$ erreicht werden.

\subsection{Abhängigkeiten der PPIs untereinander}

Die besondere Schwierigkeit bei der Auswahl der Indikatoren besteht darin, dass die Ziele der einzelnen Leistungsindikatoren voneinander abhängig sind (Abb. 9). Hohe Prozesszeiten sind unmittelbar mit hohen Prozesskosten verbunden. Ebenso verhält es sich mit der internen Termintreue: Eine niedrige Termintreue, d. h. der Anteil der Ergebnisse, die Prozesse zum vorgesehenen Termin mit Terminverzögerung liefern, hat zwangsläufig hohe Prozesszeiten und somit hohe Prozesskosten zur Folge. Eine niedrige Prozessqualität, also abgeschlossene Ergebnisse eines Prozesses mit Nachbearbeitung, verursacht höhere Prozesszeiten und somit auch höhere Prozesskosten. Die Abhängigkeiten der einzelnen Indikatoren wird unten in der Abbildung noch einmal verdeutlicht.

Auf mögliche Ursachen von Prozessineffizienzen, also zu hohe Prozesszeiten und Prozesskosten sowie zu niedrige Prozessqualität und Termintreue, wurde an anderer Stelle hingewiesen.

Es liegt in der Verantwortung des Projektmanagements bzw. des Process-Owners, die Abhängigkeiten der Indikatoren in den einzelnen Prozessen zu berücksichtigen bzw. für ein ausgewogenes Verhältnis zu sorgen.

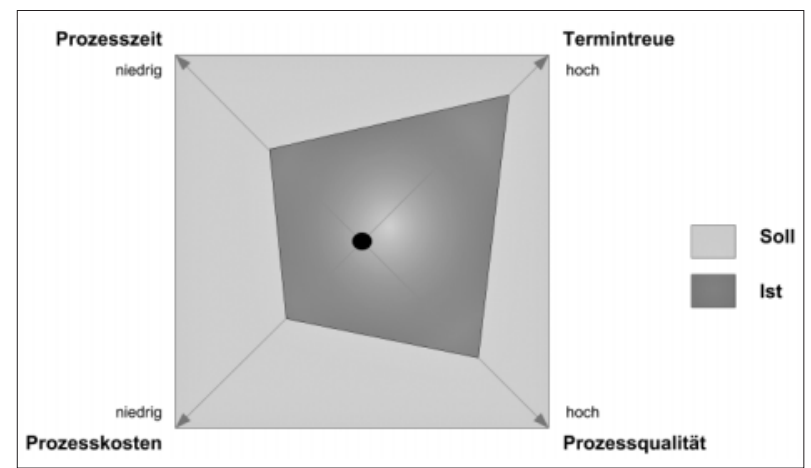

Abb. 9: PPIs der Geschäftsprozesse

\subsection{Mögliche Ursachen von Prozessineffizienzen}

Die Effizienz eines Geschäftsprozesses wird dadurch bestimmt, in welchem Maße bestimmte Leistungen wirtschaftlich erstellt werden. Wie hoch die Kosten für eine Leistungserstellung letztendlich sind, ist abhängig von der Prozesseffizienz. Die Effizienz eines Prozesses legt ferner fest, wie schnell Leistungen zur Verfügung gestellt werden können und ob sie anforderungsgerecht sind. Prozesszeiten, Prozesskosten, Prozessqualität und die interne Termintreue sind daher wichtige Messgrößen. Denn nur durch das abgestimmte Kooperieren dieser Messgrößen kann eine hohe Prozesseffizienz erreicht werden.
Die Ursachen, warum Prozesse in der Praxis nicht immer effizient arbeiten, können vielfältig sein. Im Folgenden werden mögliche Ursachen von Ineffizienzen von Prozessen aufgeführt. Mögliche Ursachen für zu lange Prozesszeiten können beispielsweise sein:

- hohe Bearbeitungszeit, Transferzeit, Wartezeit,

- überflüssige Tätigkeiten, nicht Zusammenfassen von Tätigkeiten,

- hohe Anzahl von internen Belegen/Formularen,

- häufiger Schnittstellen- und Systemwechsel,

- häufiger Wechsel von Verantwortlichkeiten und Organisationseinheiten,

- Übertragungszeiten von Daten (Server, Data Warehouse) und

- ungenügende Parallelisierung von Einzelaktivitäten.

Prozesskosten beziehen sich auf die Inanspruchnahme von Ressourcen. Mögliche Ursachen von zu hohen Prozesskosten können sein:

- Durchlaufzeiten,

- Mitarbeiter (Kostenstelle),

- Rechenleistung (CPU),

- Datenübertragung und Datenfernübertragung.

Die Prozessqualität beruht auf gemessenen Fehlern, d. h., der Prozess erfüllt die festgelegte Erwartung oder Anforderung nicht zu $100 \%$. Mögliche Ursachen für hohe Fehlerraten können sein:

- unvollständige oder ungenaue Anforderungsdefinition,

- unvollständige Dokumente und

- unvollständige Angebotserstellung.

Die Ursachen der internen Termintreue sind weit gehend mit den schon oben aufgeführten Ursachen für die Prozesszeit identisch.

\subsection{Entwicklung einer Matrix als Hilfsmittel für die Entscheidungsfindung von PPIs}

Es stellt sich nun die Frage, wie sich einzelne PPIs auf einzelne Prozesse anwenden lassen. Nicht bei allen Prozessen sind die Prozesszeiten, Prozesskosten, Prozessqualität und die interne Termintreue relevant. Wünschenswert wäre hier ein Hilfsmittel, das bei der Auswahl die Entscheidungsfindung erleichtert, ob ein Indikator wie Prozesszeit, Prozesskosten, Prozessqualität oder die interne Termintreue die Güte eines Prozesses wesentlich beeinflusst.

Um beurteilen zu können, ob ein bestimmter Indikator für einen Prozess als Messgröße relevant ist, sind folgende Überlegungen hilfreich:

- Ist der zeitliche Aspekt des Prozesses relevant?

- Lassen sich Prozesskosten durch Ressourceneinsparungen in dem Prozess reduzieren?

- Besteht die Möglichkeit der Nacharbeit von fehlerhaften Ergebnissen innerhalb des Prozesses?

- Kann eine Terminverzögerung in dem Prozess auftreten?

Aufgrund dieser Fragestellungen lässt sich eine einfache Entscheidungsmatrix als Hilfsmittel für die Entscheidungsfindung von PPIs für Prozesse generieren. In diese Matrix können die relevanten Indikatoren für einen Prozess bzw. eine Funktion auf der untersten Ebene des Prozessmodells eingetragen werden (Abb. 10). In der Tabelle steht PZ für Prozesszeit, PK für Prozesskosten, PQ für Prozessqualität und TT für interne Termintreue. 


\begin{tabular}{|l|c|c|}
\hline \multicolumn{1}{|c|}{ Indikator } & PPI & Einheit \\
\hline Relevante Fragestellungen & $\mathrm{PZ}$ & Tage \\
\hline Ist eine Reduzierung der Durchlaufzeit möglich? & $\mathrm{PK}$ & $\epsilon$ \\
\hline Ist eine Reduzierung der Ressourcen möglich? & $\mathrm{PQ}$ & $\%$ \\
\hline Ist eine Reduzierung der Nacharbeit möglich? & $\mathrm{TT}$ & $\%$ \\
\hline Ist eine Reduzierung der Terminverzögerung möglich? &
\end{tabular}

Abb. 10: Entscheidungsmatrix als Hilfsmittel für die Auswahl relevanter PPIs

\subsection{Herleitung von geschäftsprozessbezogenen Leistungsindikatoren}

Mit den oben definierten Leistungsindikatoren wurden nicht nur die Voraussetzungen geschaffen hinsichtlich der Berechnung der Indikatoren auf der horizontalen Ebene der Wertschöpfungskette, sondern auch die Grundlage für die kausalen Zusammenhänge bezüglich der vertikalen Dekompositionsebenen der Wertschöfpungskette (Abb. 11).

Neben der Aggregation der Leistungsindikatoren bzw. der Ist-Werte der Indikatoren sind natürlich auch die entsprechenden Ziel-Werte der Indikatoren in vertikaler Richtung der Wertschöpfungskette hin zu verdichten. Dabei handelt es sich nach der vorgestellten Methode um eine Bottom-Up-Vorgehensweise.

Bei termin- und kostengebundenen Projekten (Festpreisprojekte) ist diese Vorgehensweise nicht immer relevant. Projekte müssen termin- und kostengerecht ausgeführt werden, also sollten Vorgaben schon auf der obersten Ebene der Wertschöpfungskette definiert werden. In diesem Fall müssen die Ziel-Werte der Leistungsindikatoren von der obersten Ebene der Wertschöpfungskette nach unten abgeleitet werden. Dies Ableitung kann beispielsweise auf Erfahrungswerte beruhen.

\subsection{Messgrößen und Prozessziele}

Über definierte Messgrößen wird die Leistungsfähigkeit von Geschäftsprozessen gemessen. Denn erst durch diese Messgrößen lassen sich die Ist-Werte von Prozessen ermitteln. Um aber die Effizienz eines Prozesses messen zu können, sind entsprechende Zielwerte zu definieren. Nur so können Abweichungen durch den Vergleich von Zielund Ist-Werten gemessen und entsprechende Maßnahmen getroffen werden. In der folgenden Tabelle (Abb. 12) sind für die definierten Indikatoren beispielhaft die Ist- und Zielwerte dargestellt.

\begin{tabular}{|l|l|l|l|l|}
\hline \multicolumn{1}{|c|}{ Indikator } & Messgröße & \multicolumn{1}{c|}{ Ist-Wert } & \multicolumn{1}{c|}{ Ziel-Wert } & \multicolumn{1}{c|}{ Abweichung } \\
\hline Prozesszeit & PZ & 8 Tage & 5 Tage & 3 Tage \\
Prozesskosten & PK & $14.000 €$ & $12.000 €$ & $2.000 €$ \\
Prozessqualität & PQ & $79 \%$ & $92 \%$ & $13 \%$ \\
\hline Termintreue & TT & $81 \%$ & $95 \%$ & $14 \%$ \\
\hline
\end{tabular}

Abb. 12: Vergleich von Ist- und Ziel-Werten

Hinsichtlich der Abweichungen können unterschiedliche Bewertungsverfahren zum Einsatz kommen. Mithilfe von Bewertungsskalen (Abb. 13, vgl. [12]) oder so genannter Dashboards lassen sich diese visualisieren.

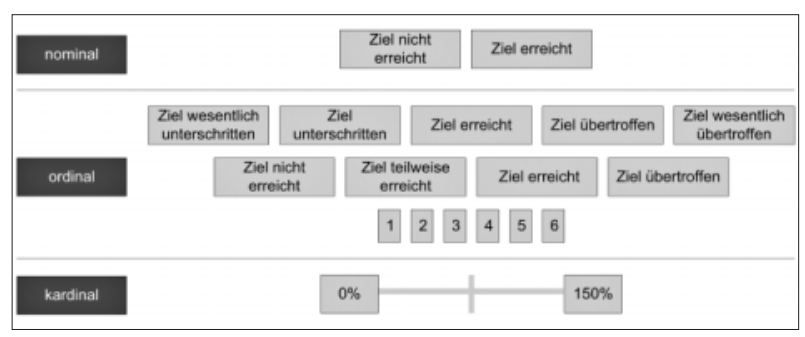

Abb. 13: Bewertungsskalen

\section{Zusammenfassung}

Es wurde eine Methodologie entwickelt, mit der sich mithilfe von Leistungsindikatoren Geschäftsprozesse analysieren und bewerten lassen. Ausgehend von den Abhängigkeiten der einzelnen Perspektiven der Balanced Scorecard, der so genannten Ursache-Wirkungs-Kette, wurde zunächst hinsichtlich dieser Perspektiven nach Leistungsindikatoren differenziert. Aufgrund dieser Differenzierung konnten Leistungsindikatoren zur Bewertung von Geschäftsprozessen, so genannte Key Performance Indicators und Indikatoren den der Geschäftstätigkeiten zugrunde liegenden Geschäftsprozesse, so genannte Process Performance Indicators, definiert werden.

Für die Prozessperspektive der Balanced Scorecard war es möglich, beispielhaft die vier Leistungsindikatoren Prozesszeit, Prozesskosten, Prozessqualität und die interne Termintreue abzuleiten. Alternativ oder als Ergänzung zu den Leistungsindikatoren könnte beispielsweise der Indikator Prozesskosten dahin gehend weiter differenziert werden, dass der Bearbeitungsaufwand der Mitarbeiter in Form von Mitarbeiterstunden in Bezug auf die jeweils fertig gestellten Ergebnisse eines Prozesses berücksichtigt wird. Als ein weiterer Indikator könnte die Zeiteffizienz oder die definierte Fehlerrate Berücksichtigung finden.

Eine besondere Schwierigkeit besteht in der Auswahl von entsprechenden Leistungsindikatoren für die Beurteilung der Effektivität und Effizienz von Geschäftsprozessen. Aus diesem Grund wurde als Hilfsmittel für die Auswahl der vier definierten Leistungsindikatoren eine Entscheidungsmatrix entwickelt.

Somit lassen sich zunächst Leistungsindikatoren auf der untersten Ebene der Wertschöpfungskette ableiten. Hinsichtlich der definierten Leistungsindikatoren wurden nicht nur die Voraussetzungen für die Berechnung der Indikatoren auf der horizontalen Dekompositionsebene der Wertschöpfungskette, sondern auch eine Basis für die kausalen Zusammenhänge der vertikalen Ebenen geschaffen. Aufgrund der somit aggregierten Leistungsindikatoren stehen auf der obersten Ebene der Wertschöpfungskette nur wenige Indikatoren zur Verfügung, um die Leistungsfähigkeit von Geschäftsprozessen beurteilen und letztendlich optimieren zu können. Denn es macht keinen Sinn, mit hunderten von Indikatoren zu arbeiten, da sich die Mitarbeiter diese Indikatoren merken müssen, um entsprechende Maßnahmen einleiten zu können, damit Geschäftsprozesse optimiert werden können.

Die vorgestellte Methodologie könnte als Grundlage dienen, um Geschäftsprozesse mit Prozessverbesserungsmethoden wie Total Cycle Time, Kaizen oder Six Sigma zu verbessern und die Prozessleistung zu steigern. 


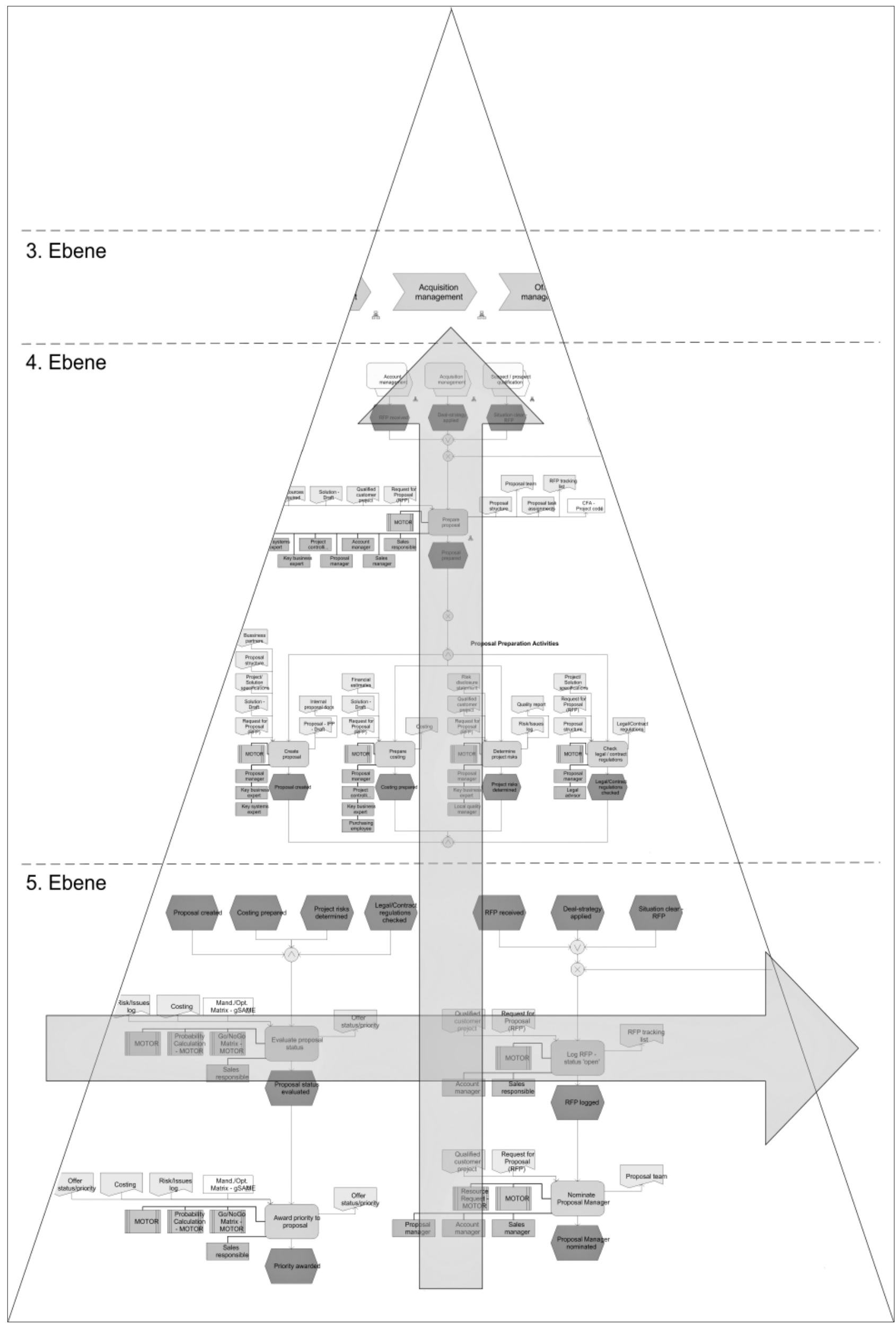

Abb. 11: Horizontale und vertikale Ausrichtung der Wertschöpfungskette 


\section{Literatur}

[1] Becker, J.; Kugeler, M.; Rosemann, M. (Hrsg.): Prozessmanagement, 4. Auflage, Springer-Verlag, Berlin, Heidelberg, New York 2003

[2] Best, E.; Weth, M.: Geschäftsprozesse optimieren, 1. Auflage, Gabler-Verlag, Wiesbaden 2003

[3] Brinkmeier, A.: Konzeption eines Kennzahlensystems zur Steuerung von IT-Dienstleistungsprozessen im Rahmen des Entwurfs eines Führungsinformationssystems, Diplomarbeit, Institut für Wirtschaftsinformatik, Technische Universität Braunschweig, Braunschweig 2004

[4] Computer Zeitung: Prozessdenken verbindet IT und Management, Computer Zeitung Nr. 47/17. November 2003, S. 12, Konradin Verlag, Leinfelden 2003

[5] Fischer, D.: Operatives Controlling: Geschäftsprozesse und Kennzahlen, Fachhochschule München, München 2004

[6] Freidinger, R.: Geschäftsprozesse im Unternehmen optimieren, Manuskript zum Vortrag Arbeitskreis Controlling, 5. Dezember 2003, Fachhochschule Pforzheim, Pforzheim 2003

[7] Fridag, H. R.; Schmidt, W.: Balanced Scorecard, 1. Auflage, Haufe Verlagsgruppe, Freiburg, Berlin, München 1999

[8] Füermann, T.; Dammasch, C.: Prozessmanagement, 2. Auflage, Carl Hanser Verlag, München, Wien 2002

[9] Deriving Enterprise-Based Measures Using the Balanced Scorecard and Goal-Driven Measurement Techniques, Technical Note CMU/SEI-2003-TN-024, 2003, www.sei.cmu.edu/ pub/documents/03.reports/pdf/03tn024.pdf

[10] gedas: Global CIO Balanced Scorecard, From Strategy to Action, gedas AG, Berlin 2004

[11] Hammer, M.; Champy, J.: Business Reengineering, CampusVerlag, Frankfurt, New York 1994

[12] Horváth, P. \& Partner (Hrsg.): Balanced Scorecard umsetzen, 3. Auflage, Schäffer-Poeschel Verlag, Stuttgart 2004

[13] James, D.: IT Performance: How the Best Are Measured, Rushmore University, Grand Cayman, USA, BWI 2002, www.dljames.com/kempis.htm

[14] Jetter, W.: Performance Management, Schäffer-Poeschel Verlag, Stuttgart 2000

[15] Kaplan, R. S.; Norton, D. P.: Balanced Scorecard, SchäfferPoeschel Verlag, Stuttgart 1997

[16] Krahn, A.; Kueng, P.; Lüthi, A.: Geschäftsprozess-Indikatoren auf der Basis von Zielen, Erfolgsfaktoren und Handlungsmöglichkeiten, Interne Publikationen Nr. 97-06, Institut für Informatik, Universität Freiburg (Schweiz), Freiburg 1997

[17] Mittag, N.: Entwicklung eines Kennzahlensystems auf Basis des prozessorientierten Managementsystems eines ITDienstleistungsunternehmens, Diplomarbeit, Technische Fachhochschule Berlin, Berlin 2004

[18] Schmelzer, H. J.; Sesselmann, W.: Geschäftsprozessmanagement in der Praxis, 3. Auflage, Carl Hanser Verlag, München, Wien 2003

[19] Staud, J.: Geschäftsprozessanalyse, 2. Auflage, Springer-Verlag, Berlin, Heidelberg, New York 2001

[20] Steinweg, C.: Management der Software-Entwicklung, 5. Auflage, Vieweg-Verlag, Wiesbaden 2004

\section{Autor}

Prof. Dr. Klaus D. Bösing

Technische Fachhochschule Wildau

Fachbereich Betriebswirtschaft/Wirtschaftsinformatik

Fachgebiet Software Engineering

Tel. +49 3375 508-952

boesing@wi-bw.tfh-wildau.de 